\title{
Publisher Correction: Pathways to eating in children and adolescents with obesity
}

\author{
Hayyah Clairman ${ }^{1,10} \cdot$ Elizabeth Dettmer ${ }^{2} \cdot$ Annick Buchholz $^{3} \cdot$ Kristina Cordeiro $^{4} \cdot$ Quazi lbrahim $^{5}$. \\ Katerina Maximova ${ }^{6}$ - Alene Toulany ${ }^{7}$ - Valerie H. Taylor $^{8}$ - Debra K. Katzman ${ }^{1,7}$ - Katherine M. Morrison ${ }^{9}$. \\ Jill Hamilton ${ }^{1,10} \cdot$ on behalf of the CANPWR Investigators
}

Published online: 19 February 2019

(c) Springer Nature Limited 2019

Correction to: International Journal of Obesity; https://doi.org/10.1038/s41366-018-0271-2; published online 19 December 2018.

In the original version of this Article the following were listed as authors; however, they should have only been referred to in the Acknowledgments section and not listed in this way:

CANPWR Investigators, Ball $\mathrm{G}^{11}$, Chanoine $\mathrm{JP}^{12}, \mathrm{Ho} \mathrm{J}^{13}$, Legault $\mathrm{L}^{14}$, Mackie $\mathrm{P}^{5}$, Thabane $\mathrm{L}^{5,15}$, Zenlea $\mathrm{I}^{16,17}$.

This has now been corrected in both the PDF and HTML versions of the Article.
Jill Hamilton

jill.hamilton@sickkids.ca

1 Institute of Medical Science, University of Toronto, Toronto, Canada

2 Department of Psychology, The Hospital for Sick Children, Toronto, Canada

3 Children's Hospital of Eastern Ontario, Ottawa, Canada

4 Department of Psychology, York University, Toronto, Canada

5 Population Health Research Institute, McMaster University, Hamilton, Canada
6 School of Public Health, University of Alberta, Alberta, Canada

7 Division of Adolescent Medicine, Department of Pediatrics, The Hospital for Sick Children, Toronto, Canada

8 Department of Psychiatry, Women's College Hospital, Toronto, Canada

9 Division of Endocrinology, Department of Pediatrics, McMaster Children's Hospital, McMaster University, Hamilton, Canada

10 Division of Endocrinology, Department of Pediatrics, The Hospital for Sick Children, Toronto, Canada 